\title{
Increased Spontaneous Central Bleeding and Cognition Impairment in APP/PS1 Mice with Poorly Controlled Diabetes Mellitus
}

\author{
Juan José Ramos-Rodriguez ${ }^{1}$ • Carmen Infante-Garcia ${ }^{1}$ • Lucia Galindo-Gonzalez ${ }^{1}$ • \\ Yaiza Garcia-Molina ${ }^{1}$ • Alfonso Lechuga-Sancho ${ }^{2}$ • Mónica Garcia-Alloza ${ }^{1}$
}

Received: 22 December 2014 / Accepted: 22 June 2015 /Published online: 9 July 2015

(C) The Author(s) 2015. This article is published with open access at Springerlink.com

\begin{abstract}
Alzheimer's disease (AD) and vascular dementia $(\mathrm{VaD})$ are the most common causes of dementia, and borderlines are blurred in many cases. Aging remains the main risk factor to suffer dementia; however, epidemiological studies reveal that diabetes may also predispose to suffer AD. In order to further study this relationship, we have induced hypoinsulinemic diabetes to APPswe/PS1dE9 (APP/PS1) mice, a classical model of AD. APP/PS1 mice received streptozotocin (STZ) ip at 18 weeks of age, when AD pathology is not yet established in this animal model. Cognition was evaluated at 26 weeks of age in the Morris water maze and the new object discrimination tests. We observed that STZinduced episodic and working memory impairment was significantly worsened in APP/PS1 mice. Postmortem assessment included brain atrophy, amyloid-beta and tau pathology, spontaneous bleeding, and increased central inflammation. Interestingly, in APP/PS1-STZ diabetic mice, we detected a shift in $A \beta$ soluble/insoluble levels, towards more toxic soluble species. Phospho-tau levels were also increased in APP/PS1-STZ mice, accompanied by an exacerbated inflammatory process, both in the close proximity to senile plaque (SP) and in SP-free areas. The presence of hemorrhages was significantly higher in APP/PS1-STZ mice, and although pericytes and endothelium were only partially
\end{abstract}

Mónica Garcia-Alloza

monica.garcia@uca.es

1 Division of Physiology, School of Medicine, Instituto de Biomoleculas (INBIO), Universidad de Cadiz, Plaza Fragela 9, 4 piso, 410, Cadiz 11003, Spain

2 Department of Radiology and Pediatrics, Universidad de Cadiz, Cadiz 11003, Spain affected, it remains possible that blood-brain barrier alterations underlie observed pathological features. Our data support the implication of the diabetic process in $\mathrm{AD}$ and $\mathrm{VaD}$, and it is feasible that improving metabolic control could delay observed central pathology.

Keywords Alzheimer's disease · Vascular dementia . Diabetes $\cdot$ Streptozotocin $\cdot$ Hemorrhage $\cdot$ Amyloid-beta

\section{Background}

Alzheimer's disease (AD) and vascular dementia (VaD) are the first and second most common causes of dementia respectively. Moreover, the borderlines between them are blurred, since in many cases, traditional AD hallmarks coexist with markers of vascular injury [1]. AD pathological features include senile plaque (SP), composed by amyloid-beta (A $\beta$ ) 40 and 42 , neurofibrillary tangles formed by abnormally phosphorylated tau, and synaptic and neuronal loss [2]. On the other hand, $\mathrm{VaD}$ is a heterogeneous disease that includes microinfarcts, small vessel ischemic disease, or microvascular injury [1].

Aging remains the main risk factor to suffer AD however, the fact that most of the cases are sporadic has favored the study of other risk factors, among which diabetes plays a relevant role. Impaired insulin secretion and resistance, as well as glucose intolerance, seem to be associated with increased risk of AD [3-5]. Insulin and insulin receptors are widely distributed in regions implicated in learning and memory, such as the cortex and hippocampus, and previous studies have reported cognition impairment in type 1 diabetic children [6,7]. Also, AD brains present lower insulin levels and higher insulin receptor density when compared to control patients (for a review, 
see [8]). It has also been shown that insulin dysregulation may interfere with $\mathrm{AD}$ neuropathological features, and insulin exacerbation of inflammatory responses may interact with $A \beta$ processing and deposition (for review, see [9, 10]). Tau phosphorylation is also severely affected when insulin levels are altered [11-13], and sequential phosphorylated residues have been characterized when insulin levels are reduced [14]. Insulin participates in neurovascular control, and therefore, it may relate metabolic alterations to $\mathrm{VaD}$ [15]. Also, vascular damage has been suggested to reduce $A \beta$ clearance along interstitial fluid drainage pathways $[16,17]$ linking diabetes, $\mathrm{AD}$ and $\mathrm{VaD}$. Following this idea, the beneficial effect of intranasal insulin on cognitive function in $\mathrm{AD}$ patients has been reported [18, 19]. In order to further explore the relationship between metabolic alterations, as insulin deprivation and derived hyperglycemia, and $\mathrm{AD}-\mathrm{VaD}$ central pathology, we have induced type 1 diabetes (T1D) to a classical model of AD. We injected STZ to APP/PS1mice before A $\beta$ deposition commences, at around 4 months of age, and we have observed a significant worsening effect of cognitive abilities, including episodic memory, by 6 months of age, when $A \beta$ deposition is established in APP/PS1 mice [20]. As previously described, tau phosphorylation was significantly increased, both in the cortex and hippocampus from APP/ PS1-STZ mice. Interestingly, a shift in $A \beta$ soluble/ insoluble levels was observed, with increased soluble, more toxic, species found in APP/PS1-STZ mice, accompanied by reduced insoluble $A \beta$ levels and deposited amyloid. Also, inflammation was exacerbated, and increased microglial activation was observed in APP/PS1-STZ mice. Moreover, we observed that hemorrhage burden was worsened in APP/PS1-STZ mice, in relevant regions for learning and memory, suggesting that alterations in the bloodbrain barrier may be responsible for the pathological features detected in APP/PS-STZ mice.

\section{Results}

\section{Metabolic Characterization}

No differences were detected in any of the parameters under study before administering STZ (18 weeks) (body weight $F_{(3}$, ${ }_{33)}=0.691, p=0.564$, glucose $F_{(3,33)}=1.49, p=0.234$, and insulin $F_{(3,33)}=0.773, p=0.518$ ) (Table 1). As it could be expected, 8 weeks after STZ treatment, we observed that metabolic parameters were significantly altered, resembling T1D with poor metabolic control. A slight reduction in body weight was observed in STZ-treated mice, although differences did not reach statistical significance $\left(F_{(3,33)}=0.691, p=0.564\right)$. Glucose levels were significantly increased in STZ-treated mice and this effect was worsened in APP/PS1-STZ mice $\left(F_{(3,28)}=60.26, * * p<0.01 \mathrm{vs}\right.$. the rest of the groups, $*^{* *} p<0.01$ vs. Wt-Sham and APP/PS1-Sham) (Table 1). Insulin levels were significantly reduced in STZ-treated mice, both Wt and APP/PS1 groups $\left(F_{(3,28)}=54.59, \dagger \dagger p<0.01\right.$ vs. WtSham and APP/PS1-Sham) (Table 1).

\section{Learning and Memory Assessment}

In order to evaluate episodic memory, we performed the new object discrimination (NOD) test as previously described [21]. To our knowledge, episodic memory in a mixed model of T1D-AD has not been assessed before. We observed an overall cognitive decline in wild-type mice treated with STZ, with comparable performance to APP/PS1-Sham treated mice, and a worsening effect was observed in APP/PS1-STZ mice. Whereas no differences were observed among groups in the "when" paradigm $\left(F_{(3,83)}=1.39, p=0.251\right)$, a slight impairment was observed in Wt-STZ, APP/PS1-Sham, and APP/ PS1-STZ groups, when compared with Wt-Sham animals $\left(F_{(3,83)}=1.39, p=0.251\right)$. On the other hand, a significant impairment was observed in the "what" paradigm, in the case of

Table 1 Metabolic characterization of STZ-treated mice

\begin{tabular}{|c|c|c|c|c|c|c|}
\hline & \multicolumn{2}{|c|}{ Body weight (g) } & \multicolumn{2}{|l|}{ Glucose (mg/dl) } & \multicolumn{2}{|l|}{ Insulin ( $\mu \mathrm{g} / \mathrm{dl})$} \\
\hline & $\begin{array}{l}18 \text { weeks } \\
\text { (before STZ) }\end{array}$ & $\begin{array}{l}26 \text { weeks } \\
\text { (after STZ }\end{array}$ & $\begin{array}{l}18 \text { weeks } \\
\text { (before STZ) }\end{array}$ & $\begin{array}{l}26 \text { weeks } \\
\text { (after STZ) }\end{array}$ & $\begin{array}{l}18 \text { weeks } \\
\text { (before STZ) }\end{array}$ & $\begin{array}{l}26 \text { weeks } \\
\text { (after STZ) }\end{array}$ \\
\hline Wt-Sham & $27.96 \pm 2.18$ & $27.98 \pm 2.03$ & $149.99 \pm 4.78$ & $150.33 \pm 4.78$ & $0.63 \pm 0.04$ & $0.57 \pm 0.01$ \\
\hline Wt-STZ & $29.27 \pm 1.81$ & $23.31 \pm 2.06$ & $135.22 \pm 8.54$ & $450.33 \pm 41.95 \dagger \dagger$ & $0.65 \pm 0.04$ & $0.23 \pm 0.03 \dagger \dagger$ \\
\hline APP/PS1-Sham & $25.75 \pm 1.98$ & $25.08 \pm 2.24$ & $125.25 \pm 10.02$ & $123.57 \pm 5.40$ & $0.60 \pm 0.02$ & $0.56 \pm 0.02$ \\
\hline APP/PS1-STZ & $28.50 \pm 1.35$ & $23.46 \pm 1.37$ & $125.50 \pm 6.97$ & $562.10 \pm 21.10^{* *}$ & $0.67 \pm 0.03$ & $0.23 \pm 0.02 \dagger \dagger$ \\
\hline
\end{tabular}

No differences were detected in any of the parameters under study before administering STZ ( 18 weeks): body weight $(p=0.564)$, glucose $(p=0.234)$, and insulin $(\mathrm{p}=0.518)$. Metabolic parameters were significantly affected at 26 weeks; after STZ treatment, glucose was increased in Wt-STZ mice, and even higher increases were observed in APP/PS1-STZ mice ( ${ }^{* *} p<0.01$ vs. the rest of the groups, $\dagger \dagger p<0.01 \mathrm{vs.} \mathrm{Wt-Sham} \mathrm{and} \mathrm{APP/PS1-Sham),} \mathrm{insulin}$ was significantly reduced in STZ-treated mice ( $\dagger \dagger p<0.01$ vs. Wt-Sham and APP/PS1-Sham), and whereas body weight was slightly reduced in STZtreated mice, differences did not reach statistical significance $(p=0.35)$. Data are representative of $6-11$ mice and differences were detected by one-way ANOVA for independent samples followed by Tukey $b$ or Tamhane tests as required 
APP/PS1-Sham and Wt-STZ animals, and a worsening effect was observed in APP/PS1-STZ-treated mice $\left(F_{(3,83)}=15.18\right.$, $*^{*} p<0.01$ vs. the rest of the groups, $+\downarrow p<0.01$ vs. Wt-Sham) (Fig. 1a). A similar profile was observed for the "where" paradigm $\left(F_{(3,83)}=27.34, * * p<0.01\right.$ vs. the rest of the groups, $+t p<0.01$ vs. Wt-Sham) (Fig. 1a).

We also evaluated spatial memory in the Morris water maze (MWM) test, and we detected a significant group $\times$ day effect along the acquisition phase, by two-way ANOVA $\left(F_{(9,465)}=1.92, * p=0.04\right)$. Further assessment of individual days revealed an overall increase in the required time to locate the platform for $\mathrm{Wt}-\mathrm{STZ}$ and APP/PS1-Sham mice, and a worsening effect in APP/PS1-STZ mice, that was especially relevant during the last day of the acquisition phase (day $1 F_{(3,124)}=$ $1.667, p=0.177$, day $2 F_{(3,124)}=7.24$, $\$+p<0.01$ vs. Wt-Sham, day $3 F_{(3,124)}=9.48$, $+\$ p<0.01$ vs. Wt-Sham, day $4 F_{(3,121)}=21.43, * * p<0.01$ vs. the rest of the groups, $+\nmid p<0.01$ vs. Wt-Sham] (Fig. 1b). Similar memory impairment was observed during the retention phase. In retention 1 ( $24 \mathrm{~h}$ after completing the acquisition phase), we observed a reduction in the time that APP/PS1-STZ mice spent in the quadrant (quadrant 2) where the platform used to be located $\left(F_{(3,26)}=4,93\right.$
$\$ p=0.008$ vs. Wt-Sham) (Fig. 1c). On the second retention phase $(72 \mathrm{~h}$ after completion of the acquisition phase), we observed that memory dysfunction was statistically significant in the Wt-STZ group, and cognitive impairment was worsened in APP/PS1-STZ animals $\left(F_{(3,24)}=45.13\right.$, $\dagger \dagger p=0.006$ vs. Wt-Sham and APP/PS1Sham, $+\$ p<0.01$ vs. Wt-Sham) (Fig. 1c).

We analyzed motor activity in all animals under study by measuring locomotor activity in the open field, and no differences among groups were observed when we compared the distances in the proximity of the walls $\left(F_{(3,25)}=1.34, p=\right.$ $0.283)$ or in the center of the arena, indicative of anxiety-like behavior $\left(F_{(3,25)}=1.33, p=0.285\right)$ (Table 2$)$. Swimming speed in the MWM also remained unaltered $\left(F_{(3,26)}=0.822, p=\right.$ $0.494)$. Similarly, final speed in the rotarod test was not affected in any of the groups under study $\left(F_{(3,24)}=1.75, p=0.183\right)$. Motor activity or anxiety-related paradigms were unaffected (Table 2), supporting that our observations in learning and memory were not due to motor function limitations. Altogether, our data suggest the presence of cognitive impairment both in AD (APP/PS1-Sham) and diabetic (Wt-STZ) mice, with a synergistic effect in APP/PS1-STZ mice, supporting a crosstalk between both pathology types that worsens episodic and spatial memory.
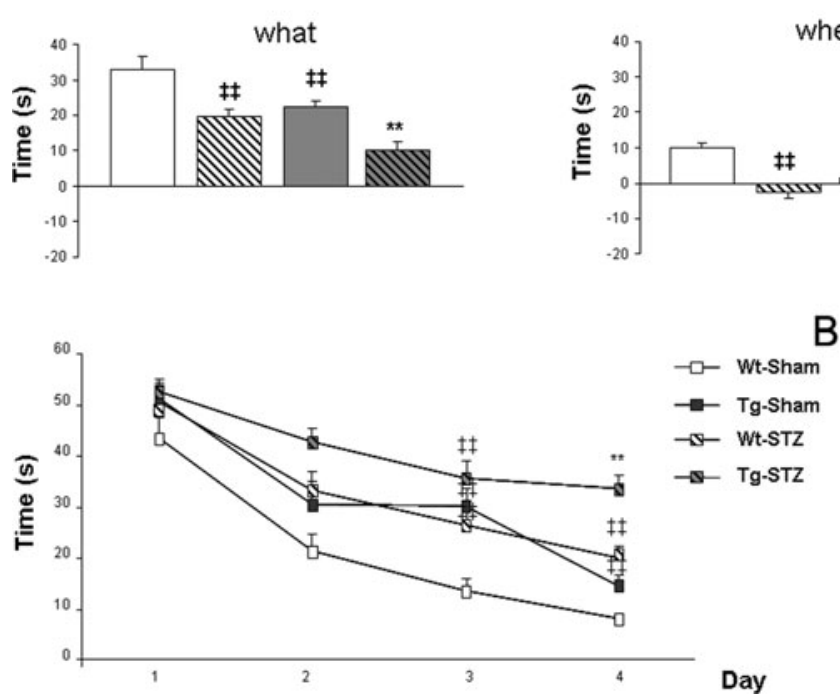

Fig. 1 Learning and memory activities are impaired in APP/PS1-STZ mice. a The NOD test revealed that episodic memory was compromised in APP/PS1-Sham and Wt-STZ mice and this effect was worsened in APP/PS1-STZ mice for "what" $(* * p<0.01$ vs. the rest of the groups, $+t p<0.01$ vs. Wt-Sham) and "where" $\left({ }^{* *} p<0.01\right.$ vs. the rest of the groups, $\$ t p<0.01$ vs. Wt-Sham), whereas differences did not reach statistical significance in the case of the "when" paradigm $(p=0.251)$. b Spatial learning and memory were also affected in the MWM test, and a significant group $\times$ day effect was detected by two-way ANOVA for independent samples $\left({ }^{*} p=0.04\right)$. Further daily assessment revealed that learning was significantly compromised along the acquisition phase in

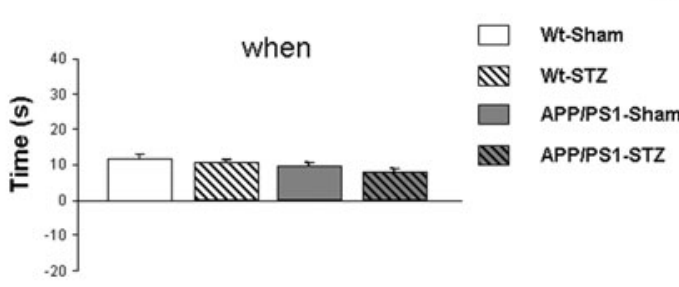

C

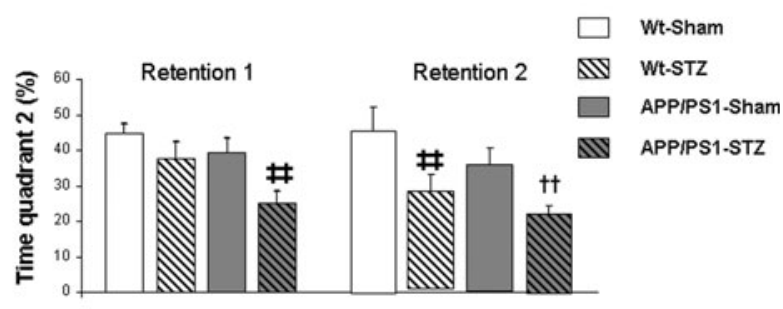

APP/PS1-Sham and Wt-STZ mice, and this effect was worsened in APP/ PS1-STZ mice (day $1 p=0.177$, day $2+p<0.01$ vs. Wt-Sham, day 3 $\$ \$ p<0.01$ vs. Wt-Sham, and day $4 * * p<0.01$ vs. the rest of the groups, $+\ddagger p<0.01$ vs. Wt-Sham). c A significant impairment was also observed in APP/PS1-STZ mice on the retention 1 ( $24 \mathrm{~h}$ after acquisition) ( $\$ p<0.01$ vs. Wt-Sham). On retention 2 (72 h after acquisition), we observed an overall worse performance in all groups; Wt-STZ mice were significantly impaired and this effect was exacerbated in APP/PS1-STZ mice $(\dagger+p<0.01$ vs. Wt-Sham and APP/PS1-Sham, $+t p<0.01$ vs. Wt-Sham). Data are representative of 6-9 mice and differences were detected by oneway ANOVA followed by Tukey $b$ or Tamhane tests as required 
Table 2 No differences in motor function were observed among groups

\begin{tabular}{lllll}
\hline & Distance of outer arena $(\mathrm{cm})$ & Distance of inner arena $(\mathrm{cm})$ & Swimming speed (cm/s) & Rotarod final speed (rpm) \\
\hline Wt-Sham & $4389.88 \pm 612.29$ & $702.47 \pm 57.64$ & $13.02 \pm 0.82$ & $23.17 \pm 2.15$ \\
Wt-STZ & $3628.93 \pm 245.72$ & $646.58 \pm 48.45$ & $11.48 \pm 0.93$ & $17.67 \pm 1.69$ \\
APP/PS1-Sham & $4709.50 \pm 510.19$ & $783.09 \pm 63.64$ & $11.19 \pm 0.58$ & $16.00 \pm 2.82$ \\
APP/PS1-STZ & $4693.93 \pm 373.25$ & $759.09 \pm 42.62$ & $11.74 \pm 0.82$ & $16.67 \pm 2.29$ \\
\hline
\end{tabular}

No differences were observed in any of the parameters under study (distance of outer arena, $p=0.283$; distance of inner arena, $p=0.285$; swimming speed, $p=0.494$; rotarod final speed, $p=0.183$ )

\section{Brain Morphology}

Brain weight was significantly reduced in STZ-treated mice and APP/PS1-STZ mice were more severely affected, although no differences were detected when compared with wild-type STZ-treated animals $\left(F_{(3,26)}=7.18, \dagger \dagger p<0.01\right.$ vs. Wt-Sham and APP/PS1-Sham) (Fig. 2a). Since brain weight is a very rough approximation to measure brain atrophy, cresyl violet staining was performed on brain hemisections and cortical and hippocampal sizes were measured along the hemisphere. We detected that hippocampal areas were slightly reduced in STZ-treated mice, although no statistical differences were observed $\left(-1.5 \mathrm{~mm}\left[F_{(3,13)}=0.780, p=0.526\right],-2.5 \mathrm{~mm}\right.$ $\left[F_{(3,14)}=1.33, p=0.303\right],-3.5 \mathrm{~mm}\left[F_{(3,14)}=21.38, p=0.289\right]$, at least at this stage of the disease (Fig. 2b)). An overall reduction in cortical size was observed in STZ-treated mice, especially evident in APP/PS1-STZ mice $\left(1.5 \mathrm{~mm}\left[F_{(3,14)}=\right.\right.$ 41.55, $p=0.244], 0.5 \mathrm{~mm}\left[F_{(3,13)}=3.30, p=0.054\right],-0.5 \mathrm{~mm}$ $\left[F_{(3,13)}=7.84, \uparrow \dagger p=0.003\right.$ vs. Wt-Sham and APP/PS1-Sham $]$,
$-1.5 \mathrm{~mm}\left[F_{(3,13)}=15.24, \dagger \dagger p<0.001\right.$ vs. Wt-Sham and APP/ PS1-Sham $],-2.5 \mathrm{~mm}\left[F_{(3,13)}=7.84, \dagger p=0.003\right.$ vs. Wt-Sham and APP/PS1-Sham], and $\left.-3.5 \mathrm{~mm}\left[F_{(3,14)}=2.64, p=0.09\right]\right)$ (Fig. 2c, d).

The specific effect of the atrophy process on neurons was also assessed, and the percentage of neuronal nuclei (NeuN)positive cells was significantly reduced in STZ-treated mice, both in wild-type and APP/PS1 mice (Fig. 2e). The percentage of NeuN-positive cells was lower in the proximity of SP, and APP/PS1-STZ mice presented even lower densities when compared with APP/PS1-Sham mice (Fig. 2e).

\section{Amyloid-Beta Pathology}

In order to determine the effect of STZ on A $\beta$ pathology, we quantified SP in the cortex and hippocampus of APP/PS1Sham and APP/PS1-STZ mice. We randomly stained wildtype sections (from Sham and STZ-treated mice), and no SPs were detected by immunohistochemistry with $4 \mathrm{G} 8$
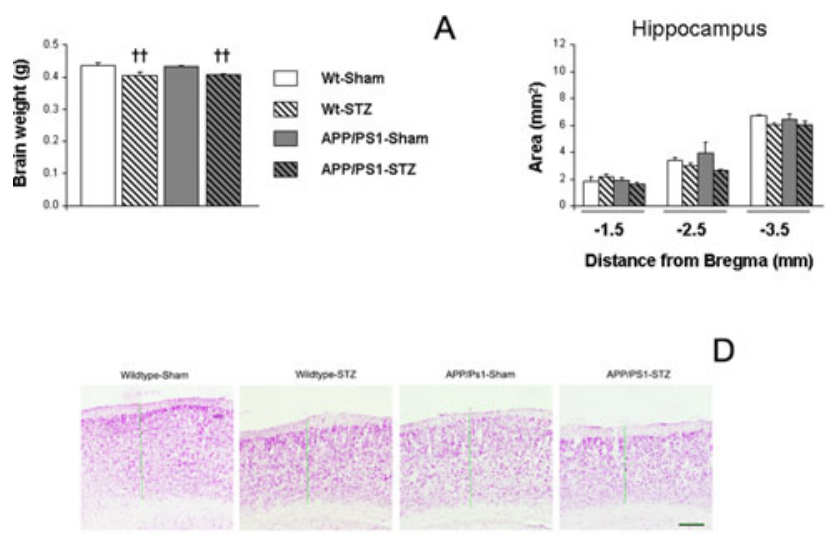

Fig. 2 Significant brain atrophy is observed after STZ administration. a We observed a reduction of brain weight in Wt-STZ and APP/PS1-STZtreated mice, at 26 weeks of age ( $\dagger \uparrow p<0.01$ vs. Wt-Sham and APP/PS1Sham). b Hippocampal assessment revealed a slight reduction in APP/ PS1-STZ-treated mice, although differences did not reach statistical significance ( $-1.5 \mathrm{~mm}, p=0.526 ;-2.5 \mathrm{~mm}, p=0.303 ;-3.5 \mathrm{~mm}, p=0.289)$. c Further assessment of cortical brain sections revealed an overall reduction of cortex size after STZ treatment, that was slightly worsened in APP/PS1 mice. Cortical size reduction reached statistical significance at $-0.5,-1.5$, and $-2.5 \mathrm{~mm}$ from bregma ( $\dagger p<0.01$ vs. wild type and APP/PS1). d Illustrative images of cortical size and thickness, stained with cresyl
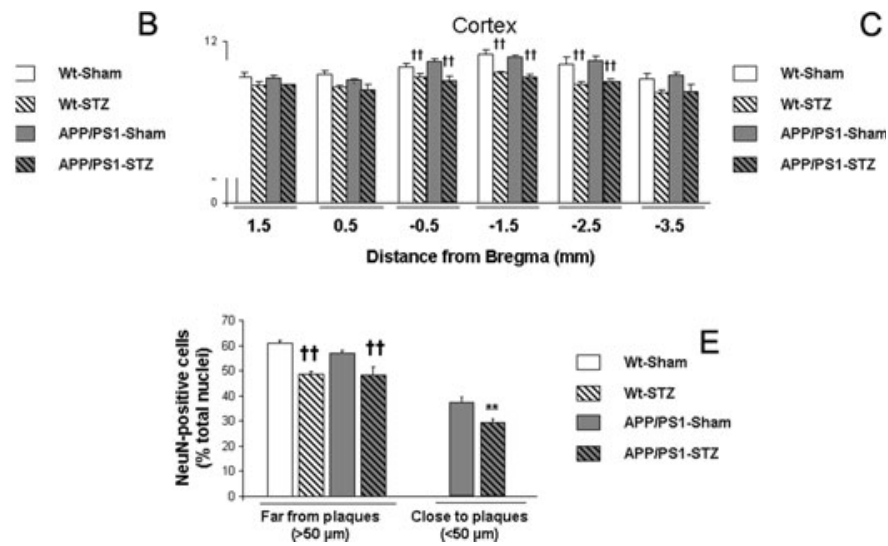

violet, in all groups under study, where significant shrinkage can be observed in STZ-treated mice. Green lines point at cortical thickness (WtSham $=1.006 \mathrm{~mm}, \mathrm{Wt}-\mathrm{STZ}=0.887 \mathrm{~mm}, \mathrm{APP} / \mathrm{PS} 1-\mathrm{Sham}=0.96 \mathrm{~mm}$, and APP/PS1-STZ $=0.825 \mathrm{~mm}$ ). Scale bar $=250 \mu \mathrm{m}$. e A significant reduction in the proportion of neurons was observed in wild-type-STZ mice far from plaques, and this effect was worsened in APP/PS1-STZ mice $\left(F_{(3,596)}=11.27, \dagger \dagger p<0.01\right.$ vs. Wt-Sham and APP/PS1-Sham). An overall reduction in proportion of NeuN-positive cells was observed in the proximity to senile plaques, and this effect was more severe in APP/PS1-STZtreated mice $(* * p=0.001$ vs. APP/PS1 Sham) 
antibody or staining with thioflavin S (TS) (Fig. 3b). SP burden was slightly reduced in the cortex from APP/PS-STZ mice after 4G8 immunohistochemistry, although differences just missed statistical significance $(p=0.052)$ (Fig. 3a, b). As in previous studies [20], the overall SP size was not altered (APP/PS1-Sham 273.84 $7.64 \mu \mathrm{m}^{2}$ and APP/PS1-STZ $\left.267.46 \pm 8.11 \mu \mathrm{m}^{2}, p=0.571\right)$, supporting the idea that SP size is stable once deposited $[17,22]$. A slight, non-significant, reduction in the number of SP per square millimeter was detected in APP/PS1-STZ-treated mice (APP/PS1-Sham 17.78 $1.23 \mathrm{SP} / \mathrm{mm}^{2}$ and APP/PS1-STZ 14.99 $\pm 1.04 \mathrm{SP} / \mathrm{mm}^{2}, p=$ 0.089). When dense-core SP burden was measured after TS staining, we observed a significant reduction of affected cortical area in APP/PS1-STZ-treated mice $(* * p<0.01$ vs. APP/ PS1-Sham) (Fig. 3a). As expected in this animal model, SP burden was lower in the hippocampus, and although we observed a similar trend to that described in the cortex, differences did not reach statistical significance when SP deposits inmunostained with $4 \mathrm{G} 8$ were quantified ( $p=0.272$ ) (Fig. 3a). Similarly, neither 4G8 deposits size (APP/PS1-Sham 232.58 \pm $15.73 \mu \mathrm{m}^{2}$ and APP/PS1-STZ 253.85 $\pm 22.62 \mu \mathrm{m}^{2}, p=0.432$ ) or number per square millimeter (/PS1-Sham $7.79 \pm 1.40 \mu \mathrm{m}^{2}$ and APP/PS1-STZ 5.29 $\pm 0.86 \mu \mathrm{m}^{2}, \mathrm{p}=0.147$ ) was affected in the hippocampus. TS deposits also reproduced this profile ( $p=$ 0.577) (Fig. 3a). We observed a similar effect when cerebral amyloid angiopathy (CAA) was quantified, and a reduction of CAA was observed in APP/PS1-STZ mice $(* p=0.022$ vs. APP/PS1-Sham) (Fig. 3c). Immunohistochemistry observations were corroborated by $\mathrm{A} \beta$ ELISA studies. As expected, $A \beta$ levels were significantly increased in APP/ PS1 mice, when compared with wild-type animals, and no differences were observed between wild-type or wild-typeSTZ-treated mice. Whereas an increase of soluble A $\beta 40$ $\left[F_{(3,10)}=39.50, * * p<0.01 \mathrm{vs}\right.$. the rest of the groups, $\dagger \dagger p<0.01$ vs. wild type and wild-type-STZ $]$ and $\mathrm{A} \beta 42\left[F_{(3}\right.$, 10) $=143.81, * * p<0.01 \mathrm{vs}$. the rest of the groups, $\uparrow \dagger p<0.01 \mathrm{vs}$. wild type and wild-type-STZ] levels was observed in the cortex from APP/PS1-STZ-treated mice (Fig. 3D), a reduction of insoluble species was observed in APP/PS1-STZ-treated mice $\left(\mathrm{A} \beta 40\left[F_{(3,9)}=61.34, * * p<0.01\right.\right.$ vs. the rest of the groups, $\dagger \uparrow p<0.01$ vs. wild type and wild-type-STZ $], \mathrm{A} \beta 40\left[F_{(3,10)}=\right.$ $22.04, * * p<0.01$ vs. the rest of the groups, $\dagger \dagger p<0.01$ vs. wild type and wild-type-STZ]) (Fig. 3d). A similar profile was observed in the hippocampus although differences only reached statistical significance when $\mathrm{A} \beta 40$ species were compared, both in the case of soluble $\left(\mathrm{A} \beta 40\left[F_{(3,10)}=81.93\right.\right.$, $* * p<0.01$ vs. the rest of the groups, $\dagger \dagger p<0.01$ vs. wild type and wild-type-STZ], $\mathrm{A} \beta 42\left[F_{(3,10)}=18.513, \dagger \dagger p<0.01\right.$ vs. wild type and wild-type-STZ]) (Fig. 3d) and insoluble $A \beta$ levels $\left(\left[F_{(3,10)}=164.24, * * p<0.01\right.\right.$ vs. the rest of the groups, $\dagger \dagger p<0.01$ vs. wild type and wild-type-STZ], A $\beta 42\left[F_{(3,10)}=\right.$ 21.93, $\dagger \dagger p<0.01$ vs. wild type and wild-type-STZ]). These data suggest a shift between soluble-insoluble $A \beta$ species in APP/PS1-STZ-treated mice that might be due to alterations in any of the steps implicated in $\mathrm{A} \beta$ production or clearance.
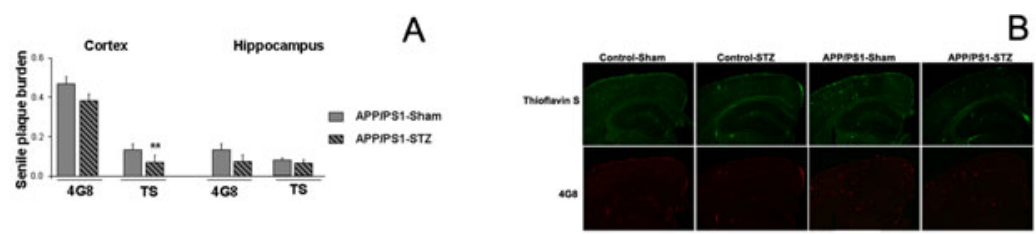

B
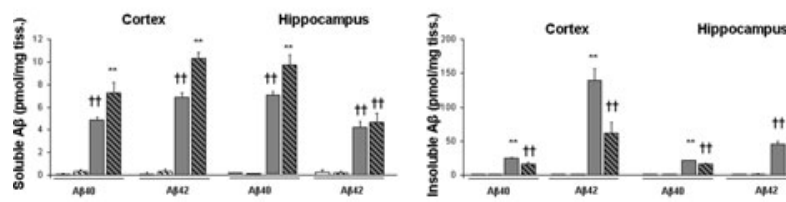

$\mathrm{D}$

Fig. 3 Cortical and hippocampal amyloid pathology after STZ treatment. a A slight reduction of cortical SP burden was observed after STZ treatment, although differences only reached statistical significance in the case of TS staining ( $4 \mathrm{G} 8 p=0.085$, TS $* * p<0.01$ vs. APP/PS1Sham). A similar profile was observed in the hippocampus although no statistical differences were detected (4G8 $p=0.272$, TS $p=0.577$ ). b Illustrative example of TS staining (green) and 4G8 (red) immunostaining in wild-type-Sham, wild-type-STZ, APP/PS1-Sham, and APP/PS1-STZ mice. Scale bar $=200 \mu \mathrm{m}$. c CAA burden was also slightly lower in APP/PS1-STZ mice $\left({ }^{*} p=0.022\right.$ vs. APP/PS1). Illustrative images of leptomeningeal vessels with CAA stained with $4 \mathrm{G} 8$. Scale $b a r=100 \mu \mathrm{m}$. d Soluble A $\beta 40$ and 42 levels were increased both in the cortex and hippocampus from APP/PS1-STZ-treated mice (cortex A $\beta 40$ $* * p<0.01$ vs. the rest of the groups, $\dagger \dagger p<0.01$ vs. wild-type-Sham and wild-type-STZ, A $\beta 42 * * p<0.01$ vs. the rest of the groups, $\dagger+p<0.01$ vs. wild-type-Sham and Wild-type-STZ; hippocampus A $\beta 40 * *<<0.01$ vs.

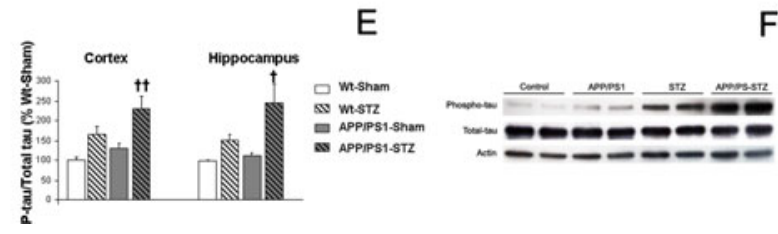

the rest of the groups, $\dagger \dagger p<0.01$ vs. wild-type-Sham and wild-type-STZ, $\mathrm{A} \beta 42 \dagger \dagger p<0.01$ vs. wild-type-Sham and wild-type-STZ). On the other hand, insoluble $A \beta 40$ and 42 levels were reduced in APP/PS1-STZ mice when compared to sham-treated animals both in the cortex $(\mathrm{A} \beta 40$, $* * p<0.01$ vs. the rest of the groups, $\dagger \dagger p<0.01$ vs. wild-type-Sham and wild-type-STZ; A $\beta 42,{ }^{* *} p<0.01$ vs. the rest of the groups, $\dagger \dagger p<0.01$ vs. wild-type-Sham and wild-type-STZ) and the hippocampus (A $\beta 40$, $* * p<0.01$ vs. the rest of the groups, $\dagger \dagger p<0.01$ vs. wild-type-Sham and wild-type-STZ; A $\beta 42, \dagger \dagger p<0.01$ vs. wild-type-Sham and wild-typeSTZ). e Phospho-tau/total tau ratio was significantly increased in APP/ PS1-STZ-treated mice both in the cortex $(\dagger \dagger p=0.003$ vs. wild-type-Sham and APP/PS1-Sham) and in the hippocampus ( $\dagger p=0.011$ vs. wild-typeSham and APP/PS1-Sham). f Illustrative example of cortical tau phosphorylation in wild-type-Sham, wild-type-STZ, APP/PS1-Sham, and APP/PS1-STZ mice 


\section{Tau Pathology}

We measured the ratio of phospho-tau/total tau levels by Western blot. A slight increase in cortical phospho-tau/total tau ratio was observed in APP/PS1-Sham and Wt-STZ mice, although differences only reached statistical significance in APP/PS1-STZ-treated mice $\left(F_{(3,26)}=5.91, \dagger \dagger p=0.003\right.$ vs. Wt-Sham and APP/PS1-Sham) (Fig. 3e, f). A similar profile was observed in the hippocampus $\left(F_{(3,27)}=4.51, \dagger p=0.011 \mathrm{vs}\right.$. Wt-Sham and APP/PS1-Sham) (Fig. 3e). Our data are in accordance with previous studies where significant alterations, in central tau phosphorylation, are observed after STZ diabetes induction $[11,14,23]$.

\section{IDE and Neprilysin}

When we analyzed central levels of enzymes implicated in insulin and $A \beta$ degradation, we did not observe any alterations in the cortex or the hippocampus from any of the groups under study (Table 3). After STZ treatment, insulin degrading enzyme (IDE) levels were unaltered in the cortex $\left(F_{(3,14)}=\right.$ $0.87, p=0.47)$ or the hippocampus $\left(F_{(3,14)}=0.054, p=0.98\right)$, and a similar outcome was observed when neprilysin levels were compared (cortex $F_{(3,14)}=10.46, p=0.709$, hippocampus $\left.F_{(3,14)}=0.54, p=0.659\right)$, suggesting that these enzymes are not affected after treatment (Table 3 ).

\section{Microglial Activation}

We analyzed the inflammatory process by quantifying microglial immunostaining with iba-1 antibody. We observed that microglial burden was slightly increased in the cortex from APP/PS1-Sham mice even far from plaques, and this effect was more pronounced in Wt-STZ-treated mice. Inflammatory process far from plaques was significantly worsened in APP/PS1-STZ-treated mice $\left(F_{(3,1382)}=42.25, * * p=0.01\right.$ vs. the rest of the groups, $\$ \ddagger p<0.01$ vs. Wt-Sham) (Fig. $4 \mathrm{a}$, c), supporting a synergistic effect between diabetes and $\mathrm{AD}$ at this level. When we analyzed microglial burden in the close proximity of SP, we also observed that the inflammatory process was favored in APP/PS1-STZ mice $(* * p<0.01$ vs. APP/PS1-Sham) (Fig. 4a, c). A similar profile was observed in the hippocampus, both far $\left(F_{(3,272)}=42.26 .45, * * p<0.01\right.$ vs. the rest of the groups, $\$ \$ p<0.01$ vs. Wt-Sham) and close to SP $\left({ }^{*} p<0.04\right.$ vs. APP/PS1-Sham) (Fig. 4b), supporting an overall increase of microglia in the hippocampus.

\section{Effect of STZ on Brain Vascular Pathology}

We observed an increase of hemorrhage burden in the cortex from STZ-treated mice, and this effect was worsened in APP/ PS1 animals $\left(F_{(3,198)}=15.81, * * p<0.01\right.$ vs. the rest of the groups, $\dagger \dagger p<0.01$ vs. Wt-Sham and APP/PS1-Sham). Individual hemorrhage size was similar among groups $\left(F_{(3}\right.$, $\left.{ }_{1002)}=0.69\right)$ although the number of hemorrhages per square millimeter was significantly increased in APP/PS1-STZ mice $\left(F_{(3,207)}=27.25, * * p<0.01\right.$ vs. the rest of the groups, $\dagger \dagger p<0.01$ vs. Wt-sham and APP/PS1-Sham) (Fig. 5a, c). These observations support a synergistic effect between diabetes and AD pathology. Hippocampal studies revealed a similar outcome to a more moderate extent, supporting the preferential affectation of the cortex (hemorrhage burden $\left[F_{(3,71)}=\right.$ 5.61, $\dagger \dagger p<0.01$ vs. Wt-sham and APP/PS1-Sham], individual hemorrhage size $\left[F_{(3,260)}=0.061\right]$, and number of hemorrhages $/ \mathrm{mm}^{2}\left[F_{(3,79)}=10.09, \dagger \dagger p<0.01\right.$ vs. Wt-sham and APP/PS1-Sham]) (Fig. 5b). Endothelial cells and pericyte densities were slightly reduced in APP/PS1-STZ mice, although differences did not reach statistical significance (endothelium $[F(3,66)=0.511, p=0.676]$, pericytes $[F(3,59)=$ $0.760, p=0.521]$ ) (Fig. 5c).

\section{Discussion}

Previous clinical and epidemiological studies support the role of metabolic disorders, including midlife hypercholesterolemia or metabolic syndrome, as risk factors to suffer AD [24-27]. It has been reported that insulin plays a relevant role in central nervous system normal activity and that alterations of insulin-dependent functions could be related to central

Table 3 Insulin degrading enzyme (IDE) and neprilysin were not affected by STZ treatment

\begin{tabular}{llllll}
\hline & \multicolumn{2}{l}{ Cortex } & & \multicolumn{2}{l}{ Hippocampus } \\
\cline { 2 - 3 } & IDE (\% Wt-Sham) & NEP (\% Wt-Sham) & & IDE (\% Wt-Sham) & NEP (\% Wt-Sham) \\
\hline Wt-Sham & $100.00 \pm 5.45$ & $100.00 \pm 8.39$ & $100.00 \pm 14.16$ & $100.00 \pm 2.96$ \\
Wt-STZ & $93.60 \pm 10.12$ & $99.94 \pm 14.00$ & $97.33 \pm 19.37$ & $110.12 \pm 11.45$ \\
APP/PS1-Sham & $79.96 \pm 17.76$ & $87.55 \pm 10.37$ & $90.46 \pm 4.62$ & $96.62 \pm 11.19$ \\
APP/PS1-STZ & $106.39 \pm 6.93$ & $106.02 \pm 10.90$ & $98.60 \pm 31.04$ & $109.76 \pm 5.57$ \\
\hline
\end{tabular}

No differences were detected when IDE and neprilysin levels were analyzed after STZ treatment in the cortex (IDE $p=0.47$, neprilysin $p=0.709$ ) or the hippocampus (IDE $p=0.98$, neprilysin $p=0.659$ ) 


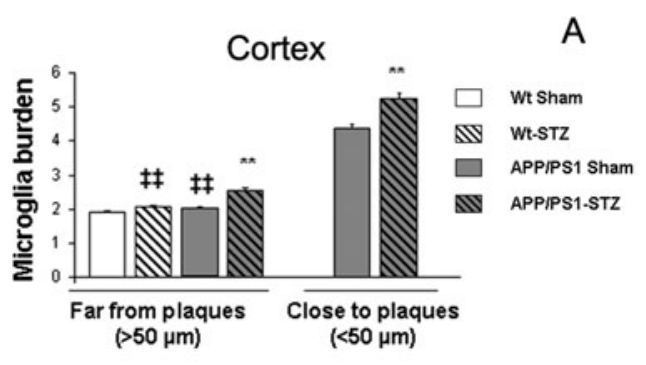

B

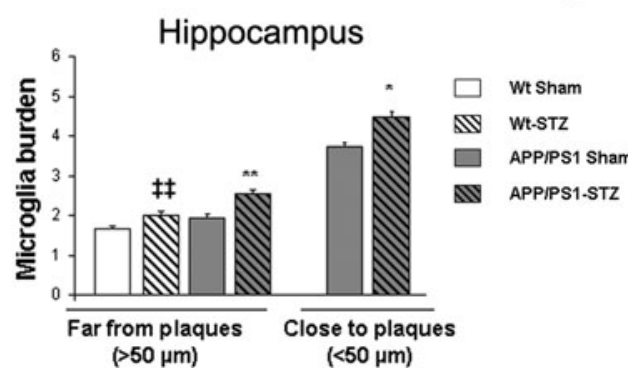

Fig. 4 Microglial activation in STZ-treated mice. a Microglial burden was significantly increased in SP-free areas in the cortex from APP/PS1Sham and Wt-STZ-treated mice. Moreover, this effect was worsened in APP/PS1-STZ-treated mice $(* * p=0.01$ vs. the rest of the groups, $\$ t p<0.01$ vs. Wt-Sham). Microglial burden in close proximity to SP was significantly higher in APP/PS1-STZ-treated mice $(* * p<0.01$ vs. APP/PS1-Sham). b A similar profile was observed in the hippocampus, where increased microglial burden in SP-free areas from Wt-STZ-treated

pathological features observed in $\mathrm{AD}$ [1, 9, 28]. Following this idea, alterations in insulin levels, such as those observed in T1D [29] and in T2D [3, 5, 30, 31], have been related to AD and $\mathrm{VaD}$. In order to further study this relationship, STZ has been widely used as an alternative model to induce sporadic $\mathrm{AD}$, by central icv administration (for review, see [32]). However, the studies on the relationship between STZ-induced diabetes and $\mathrm{AD}-\mathrm{VaD}$ alterations have been scarcer.

Our observations in the Morris water maze test are in accordance with previous studies in diabetic models [33, 34]. We detected spatial memory alterations in APP/PS1-Sham mice as well as in Wt-STZ-treated mice, and these effects were worsened in APP/PS1-STZ mice, both during the acquisition and retention phases, suggesting a synergistic effect between diabetes and AD. Previous studies have reported that STZ-induced diabetes may interfere with conditioned fear memory or induce anxiety-like behavior in rodents $[35,36]$; however, the fact that we did not observe any motor function alterations or anxiety-related behavior supports that cognitive impairment was not secondary to other central behavioral limitations. In order to further explore cognitive alterations, we explored episodic memory by a demanding approach looking at three aspects of novel object exploration "what," "where," and "when." As far as we know, episodic memory has not been assessed in this animal model before, and we found an overall significant impairment when we analyzed object,

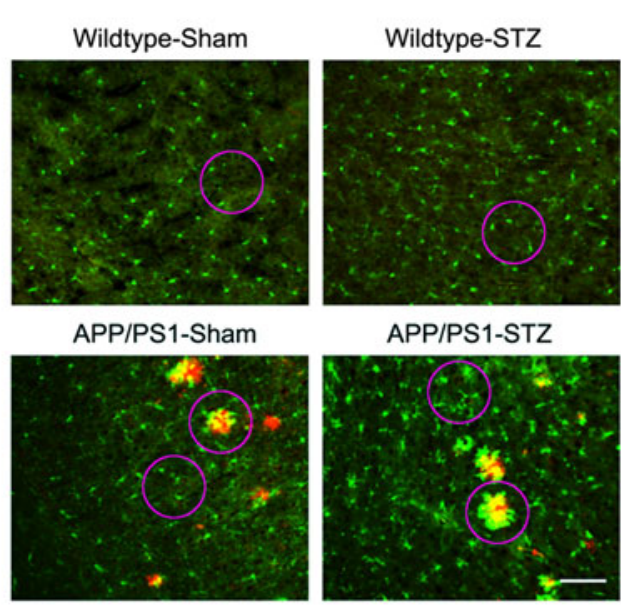

mice was worsened in APP/PS1-STZ-treated animals $(* * p<0.01$ vs. the rest of the groups, $\$+p<0.01$ vs. Wt-Sham). In the proximity of SP, microglial burden was significantly higher in APP/PS1-STZ mice $(* p<0.04$ vs. APP/PS1-Sham). c Illustrative examples of cortical microglial immunostaining using anti-microglia (iba 1, green) and anti$\mathrm{A} \beta(4 \mathrm{G} 8, \mathrm{red})$ where increased microglial burden can be detected in APP/ PS1-STZ mice, both in proximity to and far from SP. Scale bar $=125 \mu \mathrm{m}$

place, and temporal order memory in APP/PS1-STZ mice. In our hands, $\mathrm{AD}$ develops with greater certainty and severity after insulin deficiency/diabetes gets established. Previous neuropsychological studies in patients have revealed that episodic memory is early affected in the dementia process and that the diabetic process is associated with lower levels of global cognition [37]. Following this idea, general intelligence decline or vocabulary impoverishment has also been observed (for review, see [38]).

STZ treatment induced central atrophy both in APP/PS1 and wild-type mice. Brain weight was reduced and a significant reduction of cortical size was also detected. Similar observations have been previously reported in different models of diabetes [11, 39, 40]. Whereas we did not try to rescue observed abnormalities by insulin supplementation, promising effects have been reported in pilot clinical trials with $\mathrm{AD}$ patients [18]. Also, insulin replacement has been shown to successfully recover observed abnormalities in T1D mice [19] or in metabolically impaired AD transgenic mice [41], and therefore, this is a relevant line that should be further explored. Our data are in accordance with studies in T1D patients, where frontal gray matter atrophy or cortical thinning has been reported $[42,43]$. It also seems that smaller brain volumes are observed in adults with early onset of diabetes, and since T1D commonly debuts at early age, it is feasible that modifications in regular brain development may also 

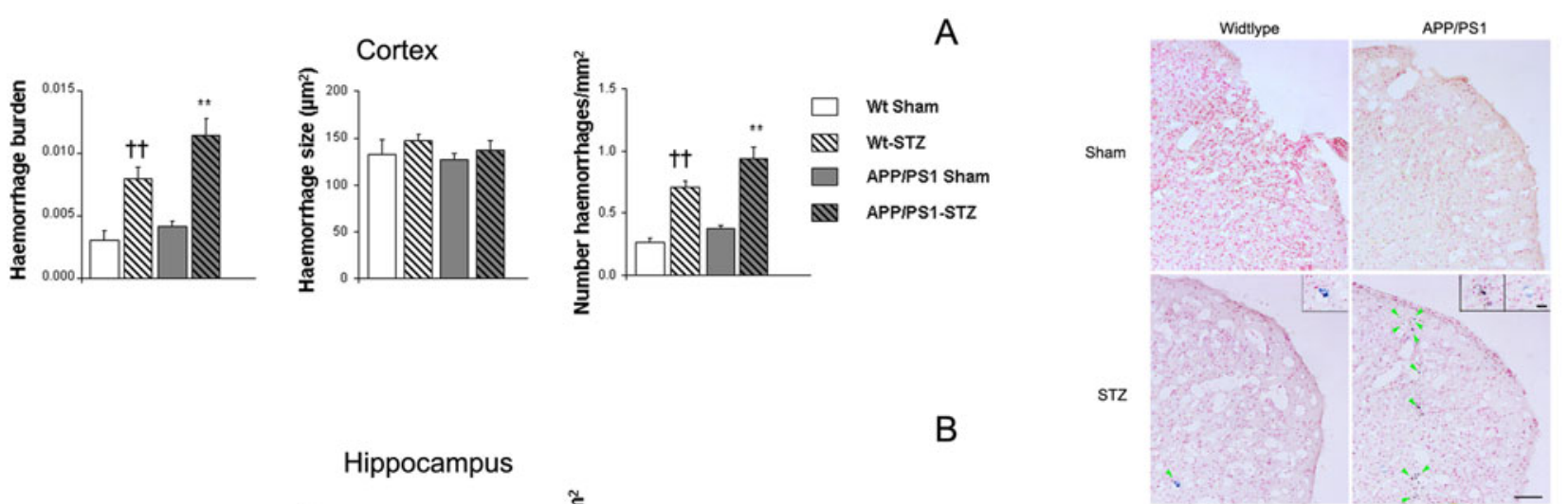

C

Hippocampus
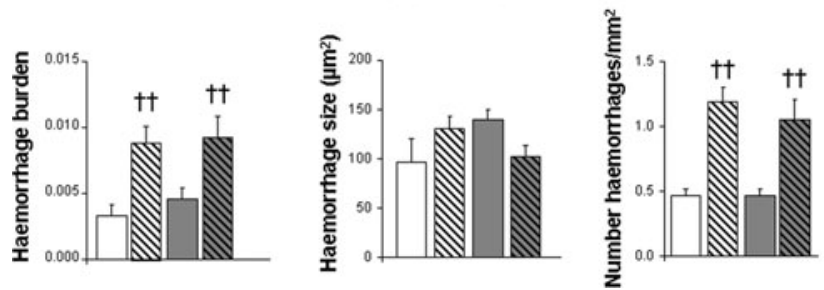

Fig. 5 Spontaneous bleeding is significantly increased in APP/PS1-STZ mice. a Hemorrhage burden was significantly increased in the cortex from Wt-STZ-treated mice and this effect was worsened in APP/PS1STZ-treated mice $(* * p<0.01$ vs. the rest of the groups, $\dagger \dagger p<0.01$ vs. Wt-Sham and APP/PS1-Sham). Individual hemorrhage size was similar in all groups $(p=0.69)$ under study, whereas a significant increase in the number of hemorrhages per square millimeter was detected in Wt-STZ mice; a worsening effect was observed in APP/PS1-STZ mice $(* * p<0.01$ vs. the rest of the groups, $\dagger \dagger p<0.01$ vs. Wt-Sham and APP/PS1-Sham). b A similar trend was detected in the hippocampus, and STZ-treated mice presented a significant increase in hemorrhage burden ( $\uparrow p<0.01$ vs. WtSham and APP/PS1-Sham), due to an increase in the number of hemorrhages per square millimeter ( $\dagger p<0.01$ vs. Wt-Sham and APP/PS1-

contribute to brain alterations observed in adulthood (for review, see [44]). However, in one-time imaging studies, it is hard to determine which area is earlier or more severely affected, and longitudinal imaging studies in T1D patients would be needed to fully understand the role of T1D in brain atrophy. We further analyzed neuronal loss in STZ mice and an overall reduction in the percentage of NeuN-positive cells was observed. We cannot obviate that STZ is toxic, and therefore, specific central action cannot be excluded; however, the synergistic effect observed in the close proximity to SP in APP/PS1-STZ mice supports a cross-talk between AD and diabetes, as previously described by other groups with similar experimental approaches (for review, see [45]).

When we analyzed AD-related central pathology, we observed a dramatic increase in the phospho-tau/total tau ratio in APP/PS1-STZ-treated mice, both in the cortex and the hippocampus. Studies on 3xTg-AD mice have shown no effect on tau phosphorylation after STZ administration [23]; however, other groups have reported similar findings to ours, in APP/ PS1 mice [46], and also increased tau phosphorylation has been observed in different diabetic models (for review, see
[10]). Moreover Clodfelder-Miller et al. have sequentially characterized affected phosphorylated tau residues after insulin depletion induced by STZ [14]. They also showed that the process leading to increased tau phosphorylation is insulin dependent, since insulin administration after short-term insulin deficiency reduced tau hyperphosphorylation at selective sites [14]. It has also been described that STZ administration may accelerate amyloid pathology in APP mice by increasing total A $\beta$ levels and SP [46]. This effect might be mediated by AGEs/RAGE/NF-kB pathway modulation [47], and enhanced APP processing has also been reported in this and other AD models [34, 48]. Surprisingly, in our hands APP/ PS-STZ-treated mice did not display increased CAA or SP deposition in the cortex or the hippocampus, although we did not specifically checked previously referred pathways, and therefore, it is feasible that APP processing or the AGEs/RAGE/NF-KB pathway could be affected. On the other hand, we are aware of the limited amount of CAA in this animal model at these early stages, which is mainly present in leptomeningeal and penetrating vessels, while we usually detect microhemorrhages deep in the parenchyma. To our 
knowledge, no previous studies have focused on different $A \beta$ species, and in our hands, STZ-induced insulin depletion slightly reduced SP and insoluble $\mathrm{A} \beta$ levels, whereas more toxic soluble $A \beta$ species were favored, as previously described in other AD models with metabolic alterations [12]. It is also possible that increased soluble $A \beta$ levels are an intermediate step in the production of compact deposits, and therefore, assessment of SP deposition at later time points may result in increased $A \beta$ deposition, as previously described [47]. Since $A \beta$ accumulation has been related to an imbalance between production and degradation or clearance of the peptide along perivascular spaces [17], it is also feasible that the observed shift between soluble and insoluble $A \beta$ levels could be due to alterations in any step of the production-elimination process. Following this idea, we analyzed IDE and neprilysin levels in our animals, since $A \beta$ and insulin degrading pathways tend to be reduced with age in AD patients. Previous studies have revealed reduced levels and activities of $A \beta$ and insulin degrading enzymes in diabetic rats [49]; however, our studies are in accordance with previous work on $5 \mathrm{xFAD}$ mice in which IDE was not affected after STZ administration [48], limiting a mechanistic approach in our study.

In order to explore other possibilities interfering in $A \beta$ elimination, we assessed the inflammatory process by analyzing microglial activation. We detected an overall increase of microglial burden in APP/PS1 and STZ mice and similar outcomes have been observed after STZ administration to other animal models [50]. Also, this effect was more severe in APP/PS1-STZ mice. We cannot exclude that earlier assessment of the inflammatory process could have shown greater differences, although mice ages and experimental times were selected depending on A $\beta$ pathology, STZ survival, and required time for T1D to fully develop. We also observed that vascular damage, induced by STZ administration, was exacerbated in APP/PS1-STZ-treated mice and hemorrhages were significantly increased in AD-diabetic mice. Whereas the reductions observed in endothelial cells and pericytes are limited, it remains possible that both added alterations may be responsible, at least in part, for observed vascular disease. Vascular alterations have been previously described [50], although to our knowledge, the presence of spontaneous bleeding has not been assessed in this ADdiabetes model, and our results suggest a synergistic effect between both pathologies. These data are in accordance with epidemiological studies showing that vascular disease is also a risk factor for dementia and that long-term hyperglycemia is associated with microvascular complications [51]. Following this idea, it has been also shown that cerebrovascular disease may worsen $\mathrm{AD}$ clinical symptoms $[52,53]$, and it remains possible that blood-brain barrier alterations may be responsible for the observed accumulation of $A \beta$ soluble species and learning and memory dysfunction.

\section{Conclusions}

Altogether, our data suggest that APP/PS1-STZ-treated mice present central alterations including exacerbated inflammation and spontaneous bleeding. These independent and additive adverse effects of STZ and APP/PS1 occurred with respect to $\mathrm{AD}$ pathology and may underlie observed cognitive dysfunction. Therefore, it remains possible that by controlling metabolic alterations, as previously suggested [19], central pathological features could also be reduced.

\section{Material and Methods}

\section{Animals}

We used APPswe/PS1dE9 mice (Jackson Laboratory, Bar Harbor, USA) as model of Alzheimer's disease in this study. In order to induce diabetes, mice were treated as previously described [11]. Mice (18 weeks old) were injected ip with STZ (40 mg/kg) for five consecutive days and animals were aged up to 26 weeks of age without insulin therapy. All experimental procedures were approved by the Animal Care and Use Committee of the University of Cadiz, in accordance with the Guidelines for Care and Use of Experimental Animals (European Commission Directive 2010/63/UE and Spanish Royal Decree RD 53/2013).

\section{Metabolic Determinations}

Body weight, postprandial blood glucose, and insulin levels were determined at 18 (before the commencement of the treatment) and 26 weeks of age as previously described [11]. Briefly, blood glucose levels were measured from nicked tails using the glucometer Optium Xceed (Abbott, UK). Blood for plasma insulin determination was collected from the tail vein into capillary tubes precoated with potassium-EDTA (Sarstedt, Nümbrecht, Germany). Blood samples were centrifuged during $10 \mathrm{~min}, 6500 \mathrm{rpm}$ at $4{ }^{\circ} \mathrm{C}$, and plasma fraction was stored at $-80{ }^{\circ} \mathrm{C}$ until processed. Plasma insulin levels were measured using ultrasensitive mouse enzyme-linked immunosorbent assay (ALPCO Diagnostics, Salem, NH, USA).

\section{MWM}

Fourteen days prior to sacrifice, learning and memory abilities were analyzed in the MWM test as previously described [11], with minor modifications. Briefly, the maze consisted of a round tank of water $(0.95 \mathrm{~m}$ in diameter) with four equal virtual quadrants indicated with geometric cues mounted on the walls. An escape platform was located $2-3 \mathrm{~cm}$ below the water surface and camouflaged with calcium carbonate to cloud the water. Water temperature was $21 \pm 1{ }^{\circ} \mathrm{C}$. A camera 
was mounted above the maze and attached to a computer and Smart software (Panlab, Spain). Testing was conducted in two phases: acquisition and retention. Acquisition consisted of four trials per day for 4 days with the platform submerged. During this phase, the platform was located in quadrant 2 . The time limit was $60 \mathrm{~s} /$ trial with an intertrial interval of $10 \mathrm{~min}$. If the animal did not find the platform, it was placed on it for $10 \mathrm{~s}$. The retention phase took place $24 \mathrm{~h}$ (retention 1) and $72 \mathrm{~h}$ (retention 2) after finishing the acquisition phase. In this phase, the submerged platform was removed, mice were allowed to swim for $60 \mathrm{~s}$, and the time that mice spent in the quadrant where the platform was previously located (quadrant 2) was recorded using SMART system (Panlab, Spain). Swimming velocity was also measured in order to detect any motor activity dysfunction that could bias the learning and memory assessment.

\section{Motor Function and NOD Task}

One day after finishing the MWM task, locomotor activity was assessed in all animals under study as previously described [54]. We measured the distance travelled by the mice for $30 \mathrm{~min}$ in a transparent rectangular box $(22-\mathrm{cm}$ long $\times 44-$ $\mathrm{cm}$ width $\times 40$-cm high) using SMART system (Panlab, Spain). In order to further assess anxiety-like behavior in our mice, distance travelled was analyzed in the proximity of the walls, as well as in the center of the boxes $(10 \mathrm{~cm}$ from the border). One day after actimetry, mice continued with the NOD test to assess their episodic memory as previously described [55]. On day 2 animals were exposed to two objects, for habituation purposes, not used again during the object exploration task on day 3 . On day 3 each mouse received two sample trials and a test trial. On the first sample trial, mice were placed into the center of the box containing three copies of a novel object (blue balls) arranged in a triangle-shaped spatial configuration and allowed to explore them for $5 \mathrm{~min}$. After a delay of $30 \mathrm{~min}$, the mice received a second sample trial with four novel objects (red cones), arranged in a quadratic-shaped spatial configuration, for $5 \mathrm{~min}$. After a delay of $30 \mathrm{~min}$, the mice received a test trial with two copies of the object from sample trial 2 (recent objects) placed in the same position and two copies of the object from sample trial 1 (familiar objects): one of them in the same position (familiar non-displaced object) and the other one in a new position (familiar displaced object). Integrated episodic memory for "what," "where," and "when" was analyzed as previously described [55]: "What" was defined as the difference in time exploring familiar and recent objects, "where" was defined as the difference in time exploring displaced and non-displaced objects, and "when" was defined as the difference between time exploring familiar non-displaced and recent nondisplaced objects. Motor function was also analyzed in the rotarod (Panlab, Spain), as previously described [56], with minor modifications. Briefly, mice were placed in the rotarod facing away the experimenter. The rod accelerated from 0 to $30 \mathrm{rpm}$ over $3 \mathrm{~min}$ and final revolutions per minute for each mice were recorded.

\section{Tissue Processing}

At the end of the NOD test, mice were sacrificed by chloral hydrate overdose $(60 \mathrm{mg} / \mathrm{kg})$. Brains were rapidly extracted and weighed. Subsequently, hemispheres were separated: The right hemisphere was dissected into the cortex and hippocampus, which were preserved at $-80^{\circ} \mathrm{C}$ until used, and the left hemisphere was immersed in $4 \%$ paraformaldehyde for 2 weeks at $4{ }^{\circ} \mathrm{C}$, to be then cut into $30-\mu \mathrm{m}$ coronal sections, for histochemical and immunohistochemical determinations.

\section{Cresyl Violet Staining}

We analyzed brain morphology by cresyl violet staining in sections selected $1 \mathrm{~mm}$ apart (from 1.5 to $-3.5 \mathrm{~mm}$ from bregma) [57]. All six sections were used to analyze cortex morphology and the last three sections were also used for hippocampal studies. Briefly, sections were mounted and dehydrated in $70 \%$ ethanol for $15 \mathrm{~min}$ before incubation in cresyl violet (Sigma, St. Louis, MO, USA) solution $0.5 \%(w /$ v) for $5 \mathrm{~min}$. Sections were washed and fixed in $0.25 \%$ acetic acid in ethanol for 7 min and subsequent $100 \%$ ethanol and xylene for $2 \mathrm{~min}$. Sections were mounted with DPX (Sigma, St. Louis, MO, USA) photographed with a Laser Olympus URFLT fluorescent microscope (Olympus, Japan). Images were acquired using MMIcellTools software. Cortex and hippocampus sizes were measured using ImageJ software.

\section{Prussian Blue Staining}

Postmortem study of hemorrhages was performed by Prussian blue iron staining and neutral red counterstain, as previously described with minor modifications [11]. Briefly, adjacent sections to the ones used for cresyl violet staining were mounted on slides and they were exposed to Prussian blue staining ( $\mathrm{HCl} 10 \%$ and potassium ferrocyanide $5 \%$ ) for $30 \mathrm{~min}$. Then, they were washed generously with water and rehydrated with 1-M phosphate buffer for $5 \mathrm{~min}$. Subsequently, sections were dehydrated in $70 \%$ alcohol and immersed in a neutral red solution $1 \%(v / w)$ for $5 \mathrm{~min}$. After washing, sections were fixed in $95 \%$ ethanol (with $1 \%$ acetic acid) and immersed in xylol for $4 \mathrm{~min}$. Sections were mounted with DPX (Sigma, St. Louis, MO, USA), coverslipped and photographed with an Olympus U-RFL Laser-U fluorescent microscope (Olympus, Japan). Cortical and hippocampal images were analyzed with the free software ImageJ. 


\section{A $\beta$ ELISA Measurements}

Soluble and insoluble $A \beta 40$ and $A \beta 42$ were quantified in the cortex and hippocampus from groups under study using colorimetric ELISA kits (Wako, Japan, A $\beta 40$ ref 294-62501 and A 342 ref 290-62601) as previously described, with minor modifications [20]. At each step, homogenization of 5$10 \mathrm{mg}$ of tissue in $50 \mathrm{ml}$ of lysis buffer with protease inhibitor cocktail (ref: 87787 and 87786, respectively, Thermo Scientific Pierce, Spain) was followed by centrifugation at 14 , $500 \mathrm{rpm}$ for $12 \mathrm{~min}$ at $4{ }^{\circ} \mathrm{C}$. Supernatants were retained for soluble $A \beta 40$ and 42 levels. The resultant pellet was then extracted with $50 \mathrm{ml}$ of $70 \%$ formic acid in distilled water and then centrifuged $10 \mathrm{~min}$ at $14,500 \mathrm{rpm}$ at $4{ }^{\circ} \mathrm{C}$, and this fraction was neutralized with $1 \mathrm{M}$ Tris (pH 11). Soluble and insoluble fractions were diluted $1: 10$ and 1:30, respectively. Standard curves were made using human $A \beta 40$ and $A \beta 42$ standards provided in the ELISA kit. Absorbance was measured spectrophotometrically at $450 \mathrm{~nm}$ (MQX200R2, Biotek instruments, Burlington VT, USA) and data were expressed as picomole per gram wet tissue.

\section{Amyloid-Beta, Microglia, Endothelium, Pericytes, and Neuronal Immunohistochemistry}

APP/PS1-STZ and APP/PS1-Sham mice were assessed postmortem for $A \beta$ burden in the cortex and hippocampus. Immunohistochemistry for $A \beta$ was performed as previously described [12] with minor modifications. PFA-fixed 30- $\mu \mathrm{m}$ sections were washed in PBS and pretreated with $70 \%$ formic acid for $10 \mathrm{~min}$, and subsequently, they were blocked in $5 \%$ normal goat serum (NGS) and $0.5 \%$ Triton-X 100 during $1 \mathrm{~h}$. Sections were immunostained with anti-A $\beta 17-24$ antibody 1:2000 (4G8, Covance, Spain) and anti-IBA1 1:1000 (Wako, Japan) in $1 \%$ NGS overnight at $4{ }^{\circ} \mathrm{C}$, followed by secondary goat anti-rabbit conjugated to Alexa 488 and goat anti-mouse to Alexa 595 (Invitrogen, USA) in $1 \%$ NGS $1 \mathrm{~h}$ at room temperature. Images were acquired using a Laser Olympus U-RFL-T fluorescent microscope (Olympus, Japan) and MMIcellTools software and analyzed with ImageJ software to quantify $\mathrm{A} \beta$ burden as well as the number and size of individual SP. CAA was also quantified as previously described [20] and results were expressed as CAA burden (\% of affected vessel). Endothelium (anti-CD31, 1:100, BD Bioscience, USA ) and pericyte (anti-NG2, 1:100, Millipore, USA) immunostaining was performed in the same conditions. Alexa Fluor anti-rat 594 and Alexa Fluor anti-rabbit 488 (Life Technologies, USA) were used as secondary antibodies, respectively, and percentage of vessel area covered by endothelial cells or pericytes was quantified with ImageJ software. Microglial activation was measured in the close proximity of plaques (up to $50 \mu \mathrm{m}$ from $\mathrm{SP}$ ) as well as far from SP $(>50 \mu \mathrm{m})$, in the case of APP/PS1-Sham and APP/PS1-
STZ-treated mice. In the case of wild-type mice, the same parameters were measured and compared with microglia far from plaques in transgenic mice. Sections selected $1 \mathrm{~mm}$ apart (from 1.5 to $-3.5 \mathrm{~mm}$ from bregma), in all animals under study, were coded and blind quantification was manually performed in the regions of interest (cortex or hippocampus) using ImageJ software. To quantify microglial burden ( $\%$ area covered), number of cells, and individual microglial size, the final analysis included between 4500-6900 cells/group in the cortex and 250-614 cells/group in the hippocampus. Contiguous sections that were used for neuronal immunostaining were incubated in anti-NeuN antibody (Chemicon) 1:200, and conjugated goat anti-mouse Alexa 594 was used as secondary antibody. Sections were washed and stained with 4',6diamidino-2-phenylindole (DAPI) $1 \mathrm{mg} / \mathrm{ml}$ (Sigma) (1:2000) and thioflavin S $0.001 \%$. The percentage of NeuN-positive cells (normalized by total cells stained with DAPI) was quantified in the proximity of SP in APP/PS1 and APP/PS1-STZ mice (up to $50 \mu \mathrm{m}$ ). Areas located far from SP were also compared with wild type and wild-type-STZ mice using Image $\mathrm{J}$ software.

\section{IDE, Neprilysin, Total Tau, and Phospho-tau Levels}

Western blot for IDE, NEP, phospho-tau, and total-tau levels was performed in fresh tissue as previously described [12]. Briefly, 5-10 mg of tissue from the cortex and hippocampus was pretreated in lysis buffer (Cell Signaling, USA) containing protease inhibitors and phosphatase inhibitors (Sigma, USA). Protein $(80 \mu \mathrm{g})$ in the case of total and phospho-tau, and $160 \mu \mathrm{g}$ in the case of IDE and NEP, was loaded and separated on $10 \%$ acrylamide-bisacrylamide gels. Proteins were transferred to PVDF membranes. Membranes were then immersed in blocking buffer (Invitrogen) for $1 \mathrm{~h}$ and incubated overnight at $4{ }^{\circ} \mathrm{C}$ with primary antibodies anti-total tau (1:1000; DAKO, Glostrup, Denmark), anti-phospho-tau (1:1000, clone AT8; Fisher Scientific, Waltham, MA, USA), anti-IDE (1:1000, N-Terminal 97-273, PC730, Millipore), anti-NEP (1:1000, ab951, Abcam), and anti- $\beta$-actin (1:2, 500,000 , Sigma). Membranes were washed and then incubated with a chemiluminescent immunodetection system for primary antibodies for mouse (AT8, anti-IDE, and anti- $\beta$-actin) or for rabbit (anti-total tau and anti-NEP) (Invitrogen, Carlsbad, USA) for $1 \mathrm{~h}$. Signal was detected using Novex AP Chemiluminescent Substrate (Invitrogen, Carlsbad, USA) and Kodak Biomax Light Film (Sigma, USA). Subsequent primary antibody incubations were preceded by stripping, using Western Blot Stripping Buffer (Fisher Scientific, Waltham, MA, USA) for $10 \mathrm{~min}$ at $37^{\circ} \mathrm{C}$ in agitation. Immunoblots were semi-quantified by measuring the optical density (OD) of each protein band on scanned film using the ImageJ software. Data were represented as percentage of wild-typeSham values. 


\section{Statistical Analysis}

Two-way ANOVA was performed to compare the acquisition phase in the MWM test. Further differences in the MWM and the NOD tests, as well as in postmortem studies (histology, immunohistochemistry, and Western blot), were determined by one-way ANOVA followed by Tukey $b$ or Tamhane tests as required. When only two groups (APP/PS1-Sham and APP/ PS1-STZ) were under study, differences were detected by Student's $t$ test for independent samples, whereas one-way ANOVA was used when more than two groups were under study. SPSS v.15 software package was used for all statistical analysis.

Acknowledgments Funding sources: Fundacion Eugenio Rodriguez Pascual (2015). Proyectos de Excelencia, Consejería de Economía, Innovación, Ciencia y Empleo (P11-CTS-7847), ISCIII-Subdirección General de Evaluación y Fomento de la Investigación and cofinanced by the European Union (Fondo Europeo de Desarrollo Regional, FEDE R) "Una manera de hacer Europa" PI12/00675 (Monica Garcia-Alloza).

Conflict of Interest The authors declare that they have no competing interests.

Open AccessThis article is distributed under the terms of the Creative Commons Attribution 4.0 International License (http:// creativecommons.org/licenses/by/4.0/), which permits unrestricted use, distribution, and reproduction in any medium, provided you give appropriate credit to the original author(s) and the source, provide a link to the Creative Commons license, and indicate if changes were made.

\section{References}

1. Craft S (2009) The role of metabolic disorders in Alzheimer disease and vascular dementia: two roads converged. Arch Neurol 66:300305

2. Serrano-Pozo A, Frosch MP, Masliah E, Hyman BT (2011) Neuropathological alterations in Alzheimer disease. Cold Spring Harb Perspect Med 1:a006189

3. Ott A, Stolk RP, Hofman A, van Harskamp F, Grobbee DE, Breteler MM (1996) Association of diabetes mellitus and dementia: the Rotterdam study. Diabetologia 39:1392-1397

4. Ott A, Stolk RP, van Harskamp F, Pols HA, Hofman A, Breteler MM (1999) Diabetes mellitus and the risk of dementia: the Rotterdam study. Neurology 53:1937-1942

5. Plastino M, Fava A, Pirritano D et al (2010) Effects of insulinic therapy on cognitive impairment in patients with Alzheimer disease and diabetes mellitus type-2. J Neurol Sci 288:112-116

6. Northam EA, Anderson PJ, Jacobs R, Hughes M, Warne GL, Werther GA (2001) Neuropsychological profiles of children with type 1 diabetes 6 years after disease onset. Diabetes Care 24:1541-1546

7. Schoenle EJ, Schoenle D, Molinari L, Largo RH (2002) Impaired intellectual development in children with type I diabetes: association with $\mathrm{HbA}(1 \mathrm{c})$, age at diagnosis and sex. Diabetologia 45:108-114

8. El Khoury NB, Gratuze M, Papon MA, Bretteville A, Planel E (2014) Insulin dysfunction and Tau pathology. Front Cell Neurosci 8:22

9. Bosco D, Fava A, Plastino M, Montalcini T, Pujia A (2011) Possible implications of insulin resistance and glucose metabolism in Alzheimer's disease pathogenesis. J Cell Mol Med 15(9): 18071821
10. Garcia-Alloza M (2014) Streptozotocin as a tool to induce central pathology and cognitive impairment in rodents. Streptozotocin: uses, mechanism of action and side effects. Nova Science Publishers, Hauppauge

11. Ramos-Rodriguez JJ, Ortiz O, Jimenez-Palomares M et al (2013) Differential central pathology and cognitive impairment in prediabetic and diabetic mice. Psychoneuroendocrinology 38:24622475

12. Ramos-Rodriguez JJ, Ortiz-Barajas O, Gamero-Carrasco C, de la Rosa PR, Infante-Garcia C, Zopeque-Garcia N, Lechuga-Sancho AM, Garcia-Alloza M (2014) Prediabetes-induced vascular alterations exacerbate central pathology in APPswe/PS1dE9 mice. Psychoneuroendocrinology 48:123-135

13. Kim B, Backus C, Oh S, Hayes JM, Feldman EL (2009) Increased tau phosphorylation and cleavage in mouse models of type 1 and type 2 diabetes. Endocrinology 150:5294-5301

14. Clodfelder-Miller BJ, Zmijewska AA, Johnson GV, Jope RS (2006) Tau is hyperphosphorylated at multiple sites in mouse brain in vivo after streptozotocin-induced insulin deficiency. Diabetes 55:3320 3325

15. Correia SC, Santos RX, Perry G, Zhu X, Moreira PI, Smith MA (2011) Insulin-resistant brain state: the culprit in sporadic Alzheimer's disease? Ageing Res Rev 10:264-273

16. Weller RO, Subash M, Preston SD, Mazanti I, Carare RO (2008) Perivascular drainage of amyloid-beta peptides from the brain and its failure in cerebral amyloid angiopathy and Alzheimer's disease. Brain Pathol 18:253-266

17. Garcia-Alloza M, Gregory J, Kuchibhotla KV et al (2011) Cerebrovascular lesions induce transient beta-amyloid deposition. Brain 134:3697-3707

18. Craft S, Baker LD, Montine TJ et al (2012) Intranasal insulin therapy for Alzheimer disease and amnestic mild cognitive impairment: a pilot clinical trial. Arch Neurol 69:29-38

19. Francis GJ, Martinez JA, Liu WQ et al (2008) Intranasal insulin prevents cognitive decline, cerebral atrophy and white matter changes in murine type I diabetic encephalopathy. Brain 131: 3311-3334

20. Garcia-Alloza M, Robbins EM, Zhang-Nunes SX et al (2006) Characterization of amyloid deposition in the APPswe/PS1dE9 mouse model of Alzheimer disease. Neurobiol Dis 24:516-524

21. Dere E, Huston JP, De Souza Silva MA (2005) Integrated memory for objects, places, and temporal order: evidence for episodic-like memory in mice. Neurobiol Learn Mem 84:214-221

22. Meyer-Luehmann M, Spires-Jones TL, Prada C et al (2008) Rapid appearance and local toxicity of amyloid-beta plaques in a mouse model of Alzheimer's disease. Nature 451:720-724

23. Li Y, Duffy KB, Ottinger MA et al (2010) GLP-1 receptor stimulation reduces amyloid-beta peptide accumulation and cytotoxicity in cellular and animal models of Alzheimer's disease. J Alzheimers Dis 19:1205-1219

24. Kivipelto M, Laakso MP, Tuomilehto J, Nissinen A, Soininen H (2002) Hypertension and hypercholesterolaemia as risk factors for Alzheimer's disease: potential for pharmacological intervention. CNS Drugs 16:435-444

25. Pappolla MA, Bryant-Thomas TK, Herbert D et al (2003) Mild hypercholesterolemia is an early risk factor for the development of Alzheimer amyloid pathology. Neurology 61:199-205

26. Razay G, Vreugdenhil A, Wilcock G (2007) The metabolic syndrome and Alzheimer disease. Arch Neurol 64:93-96

27. Muller M, Tang MX, Schupf N, Manly JJ, Mayeux R, Luchsinger JA (2007) Metabolic syndrome and dementia risk in a multiethnic elderly cohort. Dement Geriatr Cogn Disord 24:185-192

28. Garcia-Alloza M (2014) Streptozotocin as a tool to induce central pathology and cognitive impairment in rodents. In: Gauthier, E L (ed) Streptozotocin: uses, mechanism of action and side effects. Nova Science Publishers, Inc., Hauppauge, NY 
29. Cooray GK, Maurex L, Brismar T (2008) Cognitive impairment correlates to low auditory event-related potential amplitudes in type 1 diabetes. Psychoneuroendocrinology 33:942-950

30. Luchsinger JA, Tang MX, Shea S, Mayeux R (2004) Hyperinsulinemia and risk of Alzheimer disease. Neurology 63: $1187-1192$

31. Schrijvers EM, Witteman JC, Sijbrands EJ, Hofman A, Koudstaal PJ, Breteler MM (2010) Insulin metabolism and the risk of Alzheimer disease: the Rotterdam study. Neurology 75:1982-1987

32. de la Monte SM, Wands JR (2008) Alzheimer's disease is type 3 diabetes-evidence reviewed. J Diabetes Sci Technol 2:1101-1113

33. Ma LY, Zhang DM, Tang Y et al (2011) Ghrelin-attenuated cognitive dysfunction in streptozotocin-induced diabetic rats. Alzheimer Dis Assoc Disord 25:352-363

34. Wang X, Zheng W, Xie JW, Wang T, Wang SL, Teng WP, Wang ZY (2010) Insulin deficiency exacerbates cerebral amyloidosis and behavioral deficits in an Alzheimer transgenic mouse model. Mol Neurodegener 5:46

35. Ikeda H, Ikegami M, Kai M, Kamei J (2015) Cannabinoid functions in the amygdala contribute to conditioned fear memory in streptozotocin-induced diabetic mice: interaction with glutamatergic functions. Exp Neurol 269:233-241

36. Gupta D, Radhakrishnan M, Kurhe Y (2014) Ondansetron, a 5HT3 receptor antagonist reverses depression and anxiety-like behavior in streptozotocin-induced diabetic mice: possible implication of serotonergic system. Eur J Pharmacol 744:59-66

37. Arvanitakis Z, Wilson RS, Li Y, Aggarwal NT, Bennett DA (2006) Diabetes and function in different cognitive systems in older individuals without dementia. Diabetes Care 29:560-565

38. Kodl CT, Seaquist ER (2008) Cognitive dysfunction and diabetes mellitus. Endocr Rev 29:494-511

39. Devisser A, Yang C, Herring A et al (2011) Differential impact of diabetes and hypertension in the brain: adverse effects in grey matter. Neurobiol Dis 44:161-173

40. Wang JQ, Yin J, Song YF, Zhang L, Ren YX, Wang DG, Gao LP, Jing YH (2014) Brain aging and AD-like pathology in streptozotocin-induced diabetic rats. J Diabetes Res 2014:796840

41. Vandal M, White PJ, Tremblay C et al (2014) Insulin reverses the high-fat diet-induced increase in brain abeta and improves memory in an animal model of Alzheimer disease. Diabetes 63:4291-4301

42. Frokjaer JB, Brock C, Softeland E, Dimcevski G, Gregersen H, Simren M, M Drewes A (2013) Macrostructural brain changes in patients with longstanding type 1 diabetes mellitus - a cortical thickness analysis study. Exp Clin Endocrinol Diabetes 121:354-360

43. Hughes TM, Ryan CM, Aizenstein HJ et al (2013) Frontal gray matter atrophy in middle aged adults with type 1 diabetes is independent of cardiovascular risk factors and diabetes complications. J Diabetes Complicat 27:558-564
44. Biessels GJ, Reijmer YD (2014) Brain changes underlying cognitive dysfunction in diabetes: what can we learn from MRI? Diabetes 63:2244-2252

45. Gao C, Liu Y, Li L, Holscher C (2013) New animal models of Alzheimer's disease that display insulin desensitization in the brain. Rev Neurosci 24:607-615

46. Jolivalt CG, Hurford R, Lee CA, Dumaop W, Rockenstein E, Masliah E (2010) Type 1 diabetes exaggerates features of Alzheimer's disease in APP transgenic mice. Exp Neurol 223: 422-431

47. Wang X, Yu S, Hu JP, Wang CY, Wang Y, Liu HX, Liu YL (2014) Streptozotocin-induced diabetes increases amyloid plaque deposition in $\mathrm{AD}$ transgenic mice through modulating AGEs/RAGE/NFkappaB pathway. Int J Neurosci 124:601-608

48. Devi L, Alldred MJ, Ginsberg SD, Ohno M (2012) Mechanisms underlying insulin deficiency-induced acceleration of betaamyloidosis in a mouse model of Alzheimer's disease. PLoS One 7, e32792

49. Liu Y, Liu L, Lu S, Wang D, Liu X, Xie L, Wang G (2011) Impaired amyloid beta-degrading enzymes in brain of streptozotocin-induced diabetic rats. J Endocrinol Invest 34:26-31

50. Currais A, Prior M, Lo D, Jolivalt C, Schubert D, Maher P (2012) Diabetes exacerbates amyloid and neurovascular pathology in aging-accelerated mice. Aging Cell 11:1017-1026

51. Luitse MJ, Biessels GJ, Rutten GE, Kappelle LJ (2012) Diabetes, hyperglycaemia, and acute ischaemic stroke. Lancet Neurol 11: 261-271

52. Helzner EP, Luchsinger JA, Scarmeas N, Cosentino S, Brickman AM, Glymour MM, Stern Y (2009) Contribution of vascular risk factors to the progression in Alzheimer disease. Arch Neurol 66: 343-348

53. Launer LJ, Petrovitch H, Ross GW, Markesbery W, White LR (2008) AD brain pathology: vascular origins? Results from the HAAS autopsy study. Neurobiol Aging 29:1587-1590

54. Ramos-Rodriguez JJ, Pacheco-Herrero M, Thyssen D, MurilloCarretero MI, Berrocoso E, Spires-Jones TL, Bacskai BJ, GarciaAlloza M (2013) Rapid beta-amyloid deposition and cognitive impairment after cholinergic denervation in APP/PS1 mice. J Neuropathol Exp Neurol 72:272-285

55. Dere E, Huston JP, De Souza Silva MA (2005) Episodic-like memory in mice: simultaneous assessment of object, place and temporal order memory. Brain Res Brain Res Protoc 16:10-19

56. Stover KR, Campbell MA, Van Winssen CM, Brown RE (2015) Analysis of motor function in 6-month-old male and female 3xTgAD mice. Behav Brain Res 281:16-23

57. Franklin KBJ, Paxinos G (1997) The mouse brain in stereotaxic coordinates. Academic Press, San Diego 